\title{
DEK protein overexpression predicts poor prognosis in pancreatic ductal adenocarcinoma
}

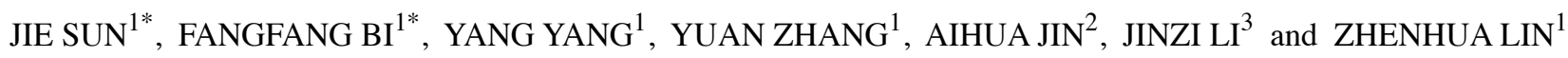 \\ ${ }^{1}$ Department of Pathology and Cancer Research Center, Yanbian University Medical College, Yanji, Jilin 133002; \\ Departments of ${ }^{2}$ Internal Medicine and ${ }^{3}$ Pediatrics, Yanbian University Hospital, Yanji, Jilin 133002, P.R. China
}

Received June 6, 2016; Accepted July 25, 2016

DOI: $10.3892 /$ or.2016.5302

\begin{abstract}
DEK, a transcription factor, is involved in mRNA splicing, transcriptional control, cell division and differentiation. Recent studies suggest that DEK overexpression can promote tumorigenesis in a wide range of cancer cell types. However, little is known concerning the status of DEK in pancreatic ductal adenocarcinoma (PDAC). Based on the microarray data from Gene Expression Omnibus (GEO), the expression levels of DEK mRNA in PDAC tissues were significantly higher than levels in the adjacent non-tumor tissues. To explore the clinical features of DEK overexpression in PDAC, 87 PDAC and 52 normal pancreas tissues were selected for immunoenzyme staining of the DEK protein. Localization of the DEK protein was detected in PANC-1 pancreatic cancer cells using immunofluorescence (IF) staining. The correlations between DEK overexpression and the clinical features of PDAC were evaluated using the Chi-squared $\left(\chi^{2}\right)$ and Fisher's exact tests. The survival rates were calculated by the Kaplan-Meier method, and the relationship between prognostic factors and patient survival was also analyzed by the Cox proportional hazard models. The expression levels of DEK mRNA in PDAC tissues were significantly higher than that in the adjacent non-tumor tissues. The DEK protein showed a primarily nuclear staining pattern in PDAC. The positive rate of the DEK protein was $52.9 \%(46 / 87)$ in PDAC, which was significantly higher than that in the adjacent normal pancreatic tissues $(7.7 \%, 4 / 52)$. DEK overexpression in PDAC was correlated with tumor size, histological grade,
\end{abstract}

Correspondence to: Dr Zhenhua Lin, Department of Pathology and Cancer Research Center, Yanbian University Medical College, Yanji, Jilin 133002, P.R. China

E-mail: zhlin720@ybu.edu.cn

Dr Jinzi Li, Department of Pediatrics, Yanbian University Hospital, Yanji, Jilin 133002, P.R. China

E-mail: yjzli@ybu.edu.cn

*Contributed equally

Key words: pancreatic ductal adenocarcinoma, DEK, survival analysis, biomarker tumor-node-metastasis (TNM) stage and overall survival (OS) rates. In addition, multivariate analysis demonstrated that DEK overexpression was an independent prognostic factor along with histological grade and TNM stage in patients with PDAC. In conclusion, DEK overexpression is associated with PDAC progression and may be a potential biomarker for poor prognostic evaluation in PDAC.

\section{Introduction}

Pancreatic cancer (PC), a highly malignant digestive system tumor, is the fourth major cause of cancer-related deaths worldwide (1). Pancreatic ductal adenocarcinoma (PDAC) is the most aggressive PC, and accounts for $>80 \%$ of PC cases. Despite continuous progress in diagnosis and treatment in recent decades, PDAC remains a great clinical challenge due to its dismal prognosis (2-7). Currently, the key obstacle to progress is the lack of accurate and specific targets for the early diagnosis of PDAC (8-10). Therefore, the identification of novel biomarkers and development of new therapeutic approaches are of great value for PDAC.

The oncoprotein DEK was initially discovered as a fusion protein with CAN/NUP214 nucleoporin due to the $(6 ; 9)$ (p23;q64) translocation in a subset of acute myeloid leukemia (AML) $(11,12)$. Now, it is emerging as a class of DNA topology modulators encoded by a gene located at chromosome $6 \mathrm{p} 22.3$ (13). The functions of DEK involve DNA supercoiling, mRNA splicing, DNA damage repair, transcriptional control and cell viability in cell progression and metabolism (14-17). As an architectural chromatin protein, DEK has been detected in numerous human malignancies including glioblastoma (18), AML (19), bladder cancer (20) and hepatocellular carcinoma (21). Khodadoust et al showed that the level of DEK expression can distinguish benign nevi from malignant melanomas, indicating that this protein may be highly useful for differentiating diagnoses (12).

Our previous study found that DEK was significantly expressed in patients with colorectal cancer, and this overexpression was associated with poor prognostic factors (22). We also revealed that the level of DEK expression was significantly increased in various solid tumors, such as breast and gastric cancer using immunohistochemical (IHC) staining $(23,24)$. However, to date, the detailed role of DEK overexpression in PDAC remains unclear. Therefore, 
we identified the clinical features correlated with DEK overexpression and the potential prognostic value of DEK in PDAC. The results revealed a significant increase in DEK expression in PDAC tissues compared to levels in the normal pancreas tissues. These findings suggest that DEK overexpression may be an independent reliable biomarker for poor prognosis in patients with PDAC.

\section{Materials and methods}

Ethics statement. The present study complied with the Helsinki Declaration and was approved by the Human Ethics Committee and the Research Ethics Committee of Yanbian University Medical College. Patients were informed that the resected specimens were stored by the hospital and potentially used for scientific research, and that their privacy may be maintained. Follow-up survival data were retrospectively collected through medical-record analyses.

Clinical samples. A total of 139 samples of pancreas tissues, including 87 PDAC and 52 adjacent normal pancreas tissues, were collected from the Tumor Tissue Bank of Yanbian University Medical College. All tissues were routinely fixed in $10 \%$ buffered formalin and embedded in paraffin blocks. The institutional Review Board of Yanbian University Medical College approved the study protocol. The pathological parameters, including gender, age, tumor location, tumor size, grading, clinical tumor-node-metastasis (TNM) stage, perineural invasion status, lymph node metastasis and survival data, were carefully reviewed for all 87 PDACs. The male to female ratio was $48: 39$, and 52 cases were $<50$ years and 35 cases were $\geq 50$ years (median age of 59 years). Tumors were located in the head of the pancreas in 59 cases, and in the body and tail of the pancreas in 28 cases. Of the 87 PDACs, 48 cases had tumor size $<3 \mathrm{~cm}$ and 39 cases had tumor size $\geq 3 \mathrm{~cm}$ (mean size of $3.36 \mathrm{~cm}$ ). In regards to the grading of PDAC, 25 cases were grade 1, 34 cases were grade 2, and 28 cases were grade 3 . Concerning the clinical TNM stage, 53 cases were stage I-II and 34 cases were TNM stage III-IV. Clinicopathological classification and staging were assessed according to the staging system established by the American Joint Committee on Cancer (AJCC). In addition, 42 cases had perineural invasion, and 45 cases had no perineural invasion; 42 cases had lymph node (LN) metastasis, and 45 cases had no LN metastasis. The normal pancreases were obtained from the resection margins of radical specimens of PDAC.

A total of 87 patients with PDAC had received surgical treatment, but not adjuvant chemotherapy at the time of data collection. The survival information of the patients was successfully collected during 30 months or until death.

Immunofluorescence (IF) staining analysis. Human PC cell line PANC-1 was obtained from the Cell Bank of the Chinese Academy of Medical Science (Shanghai, China). The cells were grown and cultured in Dulbecco's modified Eagle's medium (DMEM) (Gibco, Gaithersburg, MD, USA) supplemented with $10 \%$ fetal bovine serum and $1 \%$ penicillin/streptomycin in humidified $5 \% \mathrm{CO}_{2}$ at $37^{\circ} \mathrm{C}$.

PANC-1 cells were grown on coverslips to $70-80 \%$ confluency, and fixed with $4 \%$ paraformaldehyde for $10 \mathrm{~min}$ and

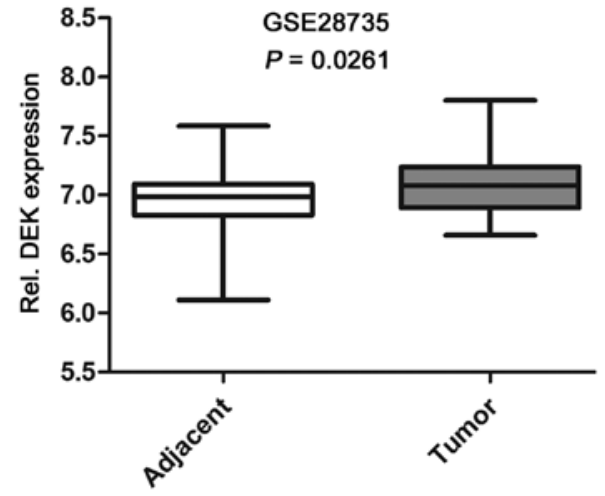

Figure 1. DEK mRNA overexpression in PDAC tissues. The analysis of DEK mRNA levels in adjacent and tumor samples from the GSE28735 dataset. Box plots showing the elevated expression of DEK during tumorigenesis in PDAC datasets. Units for $y$-axis are absolute expression value from the microarray data. PDAC, pancreatic ductal adenocarcinoma.

permeabilized with $0.5 \%$ Triton X-100 for $10 \mathrm{~min}$ at room temperature. Subsequently, after blocking with 3\% albumin bovine V (A8020; Solarbio, Beijing, China) for $1 \mathrm{~h}$, the cells were gently washed with phosphate-buffered saline (PBS). A primary antibody against DEK (1:50; 610948; BD Biosciences, Franklin Lanes, NJ, USA) was incubated with the cells at $4^{\circ} \mathrm{C}$ overnight, followed by incubation with Alexa Fluor ${ }^{\circledR} 568$ goat anti-mouse IgG (H + L) (1:1,000; A11004; Invitrogen, Carlsbad, CA, USA) for $1 \mathrm{~h}$. Then, the cells were washed with PBS and counterstained with 4',6-diamidino-2-phenylindole (DAPI) (C1006; Beyotime, Beijing, China). The coverslips were mounted with Antifade Mounting Medium (P0126; Beyotime). Finally, IF signals were visualized and recorded using a Leica SP5 II confocal microscope.

Immunoenzyme staining analysis. Immunoenzyme staining was performed using the standard streptavidin-peroxidase (SP) method. Briefly, all tissue sections were deparaffinized, rehydrated and incubated with $3 \% \mathrm{H}_{2} \mathrm{O}_{2}$ in methanol for $15 \mathrm{~min}$ at room temperature. Subsequently, the antigen was retrieved in $0.01 \mathrm{M}$ sodium citrate buffer ( $\mathrm{pH}$ 6.0). The slides were incubated with a primary antibody against DEK (1:50; 610948; BD Biosciences) at $4^{\circ} \mathrm{C}$ overnight. After incubation with biotinylated secondary antibody at room temperature for $30 \mathrm{~min}$, the slides were covered with SP complex at room temperature for $30 \mathrm{~min}$. Immunostaining was developed using 3,3'-diaminobenzidine and counterstaining with Mayer's hematoxylin. Mouse IgG isotope was used as the control and the result was negative. Furthermore, the positive tissue sections were processed as negative controls by omitting the primary antibody.

Two pathologists (Y. Yang and F. Bi) independently evaluated all tissue specimens without knowledge of the clinical data. In case of discrepancies, a final score was established by reassessment on a double-headed microscope. The scoring system for the interpretation criteria was previously described (22). Briefly, staining intensity of the tissue sections was scored as '-' for no staining, '+' was defined as weak staining, and ' ++ ' was considered as intense staining, respectively. The staining area was scored as follows: '-' (negative, no or $<5 \%$ positive cells), '十' (5-50\% positive cells), ' ++ ' ( $>50 \%$ positive cells). 


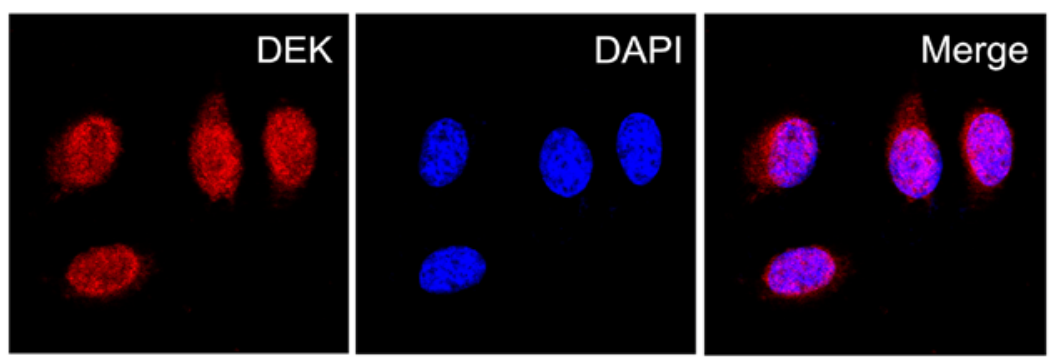

Figure 2. Immunofluorescence (IF) staining for DEK protein in the PC PANC-1 cells. The DEK protein is mainly located in the nucleus of the PANC-1 cells (red indicates DEK staining and blue indicates DAPI). PC, pancreatic cancer.

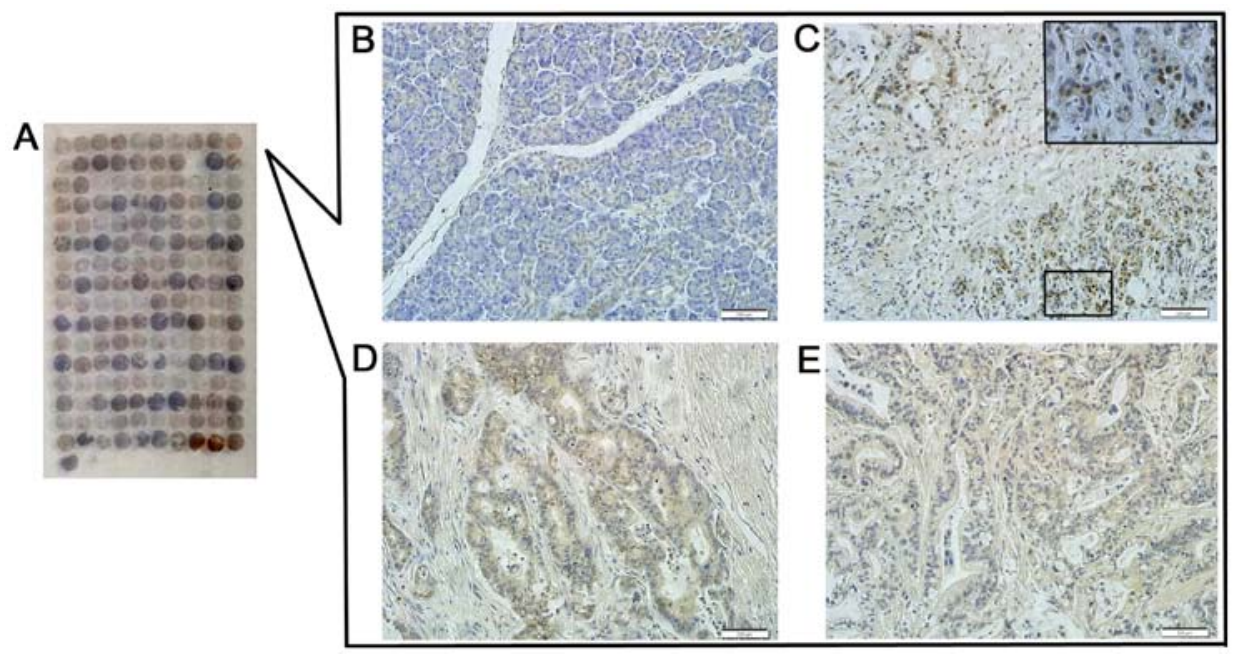

Figure 3. Immunoenzyme staining in PDAC tissue array samples. (A) Immunoenzyme staining of PDAC tissue microarray. (B) DEK protein is negative in the normal pancreas (original magnification, x200). (C) DEK protein is positive in the PDAC, and is principally localized in the nucleus of cells (original magnification, $\mathrm{x} 200$ ). (D) Weak positive staining for DEK protein is seen in the PDAC nucleus of cells (original magnification, $\mathrm{x} 200$ ). (E) DEK protein is absolutely negative in PDAC (original magnification, x200). PDAC, pancreatic ductal adenocarcinoma.

For the double scoring system together, '++' scored samples were considered as DEK overexpression, and '-' or '+' scored samples were considered as DEK low-expression.

Statistical analysis. Statistical analyses were conducted using SPSS 17.0 software (SPSS, Inc., Chicago, IL, USA). DEK mRNA expression data were obtained from GEO database. Correlations between DEK protein expression and clinicopathological features were evaluated by Chi-squared $\left(\chi^{2}\right)$ and Fisher's exact tests. The survival curves were performed using the Kaplan-Meier method, and significant differences were assessed by log-rank tests. Multivariate survival analysis was performed on all significant characteristics measured by univariate survival analysis with the Cox proportional hazard regression model. A P-value $<0.05$ was considered to indicate a statistically significant result.

\section{Results}

DEK expression in PDAC. Based on the data from Gene Expression Omnibus (GEO), we found that the expression level of DEK mRNA in PDAC tissues was significantly higher than that in the adjacent non-tumor tissues (Fig. 1). To explore the role of DEK protein in PDAC, we then determined the localization of DEK protein expression in PDAC PANC-1 cells via IF staining, and assessed the expression levels of DEK protein in PDAC and the normal pancreas tissues via immunoenzyme staining. The DEK protein showed a strictly nuclear staining pattern in PDAC (Figs. 2 and 3). Simultaneously, the positive rate of the DEK protein was 52.9\% (46/87) in PDAC, which was significantly higher than in the adjacent normal pancreatic tissues $(7.7 \%, 4 / 52)(\mathrm{P}<0.01)$. Similarly, the strong positive rate of the DEK protein was also higher in PDAC $(13.8 \%, 12 / 87)$ compared with the adjacent normal pancreatic tissues $(0 \%, 0 / 52)(\mathrm{P}<0.01)$ (Table I).

Correlations between DEK protein overexpression and clinical features of PDAC. To evaluate the role of the DEK protein in PDAC progression, we analyzed the correlation between DEK overexpression and clinicopathological features of the PDAC patients. Generally, DEK overexpression was significantly correlated with tumor size, TNM stage and grade of PDAC, but not related to gender, age, tumor location, perineural invasion status and lymph node metastasis of patients with PDAC $(\mathrm{P}>0.05)$.

The positive rate of the DEK protein was significantly higher in PDAC cases with $\geq 3 \mathrm{~cm}$ tumor size $(71.8 \%, 28 / 39)$ than in patients with $<3 \mathrm{~cm}$ tumor size $(37.5 \%, 18 / 48)(\mathrm{P}<0.01)$. For TNM clinical stage, the positive rate of DEK protein in the advanced stage (III-IV) PDAC cases was $73.5 \%(25 / 34)$, 
Table I. DEK protein expression in the PDAC cases.

\begin{tabular}{|c|c|c|c|c|c|c|}
\hline \multirow[b]{2}{*}{ Diagnosis } & \multirow{2}{*}{$\begin{array}{l}\text { No. of } \\
\text { cases }\end{array}$} & \multirow{2}{*}{$\begin{array}{c}\text { Negative } \\
\text { cases } \\
-\end{array}$} & \multicolumn{2}{|c|}{ Positive cases } & \multirow{2}{*}{$\begin{array}{c}\text { Positive rate } \\
(\%)\end{array}$} & \multirow{2}{*}{$\begin{array}{c}\text { Strongly positive } \\
\text { rate }(\%)\end{array}$} \\
\hline & & & + & ++ & & \\
\hline PDACs & 87 & 41 & 34 & 12 & $52.9^{\mathrm{a}}$ & $13.8^{\mathrm{a}}$ \\
\hline Normal pancreas & 52 & 48 & 4 & 0 & 7.7 & 0 \\
\hline
\end{tabular}

Positive rate, percentage of positive cases with + and ++ staining score; strongly positive rate, percentage of positive cases with ++ staining score; ${ }^{\text {a }}<0.01$ compared with normal pancreatic tissues. PDACs, pancreatic ductal adenocarcinomas.

Table II. Correlation of DEK protein expression and the clinicopathological features of PDAC.

\begin{tabular}{|c|c|c|c|c|}
\hline Variables & $\begin{array}{c}\text { No. of } \\
\text { cases }\end{array}$ & $\begin{array}{l}\text { DEK-positive } \\
\text { cases }(\%)\end{array}$ & $\chi^{2}$ & P-value \\
\hline Gender & & & 0.355 & 0.551 \\
\hline Male & 48 & $24(50.0)$ & & \\
\hline Female & 39 & $22(56.4)$ & & \\
\hline Age (years) & & & 1.194 & 0.275 \\
\hline$<50$ & 52 & $25(48.1)$ & & \\
\hline$\geq 50$ & 35 & $21(60.0)$ & & \\
\hline Location & & & 0.464 & 0.496 \\
\hline Head & 59 & $32(54.2)$ & & \\
\hline Body and tail & 28 & $13(46.4)$ & & \\
\hline Tumor size $(\mathrm{cm})$ & & & 10.156 & $0.001^{\mathrm{a}}$ \\
\hline$<3$ & 48 & $18(37.5)$ & & \\
\hline$\geq 3$ & 39 & $28(71.8)$ & & \\
\hline $\begin{array}{l}\text { Histological } \\
\text { grade }\end{array}$ & & & 5.993 & $0.050^{\mathrm{a}}$ \\
\hline Grade 1 & 25 & $10(40.0)$ & & \\
\hline Grade 2 & 34 & $16(47.1)$ & & \\
\hline Grade 3 & 28 & $20(71.4)$ & & \\
\hline TNM stage & & & 9.557 & $0.002^{\mathrm{a}}$ \\
\hline Stage I-II & 53 & $21(39.6)$ & & \\
\hline Stage III-IV & 34 & $25(73.5)$ & & \\
\hline $\begin{array}{l}\text { Perineural } \\
\text { invasion }\end{array}$ & & & 0.116 & 0.733 \\
\hline Absent & 45 & $23(51.1)$ & & \\
\hline Presence & 42 & $23(54.8)$ & & \\
\hline LN metastasis & & & 0.900 & 0.343 \\
\hline Negative & 45 & $26(57.8)$ & & \\
\hline Positive & 42 & $20(47.6)$ & & \\
\hline
\end{tabular}

a Significant difference. PDAC, pancreatic ductal adenocarcinoma; TNM, tumor-node-metastasis; LN, lymph node.

but only $39.6 \%(21 / 53)$ in the early stage (I-II) cases $(\mathrm{P}<0.05)$. Moreover, the positive rate of DEK was significantly higher in grade $3(71.4 \%, 20 / 28)$ than in grade $2(47.1 \%, 16 / 34)$ and grade 1 cases $(40.0 \%, 10 / 25)(\mathrm{P}<0.05)$ (Table II and Fig. 4).
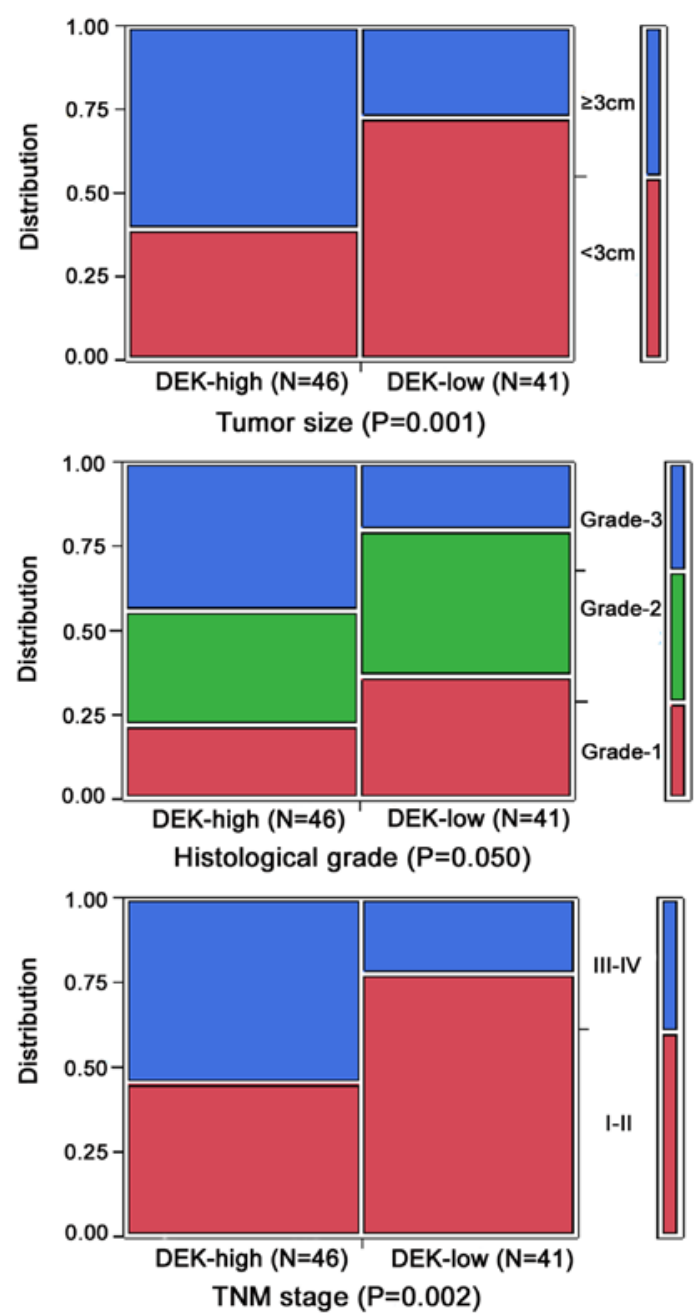

Figure 4. Relationship between DEK expression and clinicopathological significance of PDACs. DEK overexpression rates in PDAC cases with large tumor size, high histological grade, and advanced clinical stage are significantly higher than in the contrasting groups.

DEK overexpression is an independent prognostic biomarker of PDAC. To evaluate the role of DEK overexpression in PDAC progression, we analyzed the prognostic factors and overall survival (OS) in 87 PDAC cases using the Cox proportional hazards model. Univariate analysis showed that tumor size ( $\mathrm{P}=0.034)$, histological grade $(\mathrm{P}=0.000)$, TNM stage $(\mathrm{P}=0.000)$, perineural invasion status $(\mathrm{P}=0.034), \mathrm{LN}$ metastasis $(\mathrm{P}=0.004)$ and the level of DEK expression $(\mathrm{P}=0.000)$ were associated 
Table III. Univariate and multivariate survival analyses of the clinicopathological features in 87 PDAC cases.

\begin{tabular}{|c|c|c|c|c|c|c|c|}
\hline \multirow[b]{2}{*}{ Characteristics } & \multirow[b]{2}{*}{ B } & \multirow[b]{2}{*}{ SE } & \multirow[b]{2}{*}{ Wald } & \multirow[b]{2}{*}{ HR } & \multicolumn{2}{|c|}{$95 \% \mathrm{CI}$} & \multirow[b]{2}{*}{ P-value } \\
\hline & & & & & Lower & Upper & \\
\hline \multicolumn{8}{|c|}{ Univariate survival analyses } \\
\hline Gender & 0.278 & 0.229 & 1.477 & 1.320 & 0.843 & 2.066 & 0.224 \\
\hline Age & 0.278 & 0.230 & 1.457 & 1.320 & 0.841 & 2.071 & 0.227 \\
\hline Location & 0.190 & 0.237 & 0.643 & 0.827 & 0.520 & 1.315 & 0.423 \\
\hline Tumor size & 0.479 & 0.226 & 4.508 & 1.614 & 1.038 & 2.512 & $0.034^{\mathrm{a}}$ \\
\hline Histological grade & 0.734 & 0.182 & 16.261 & 2.083 & 1.458 & 2.976 & $0.000^{\mathrm{b}}$ \\
\hline TNM stage & 1.173 & 0.241 & 23.629 & 3.233 & 2.014 & 5.189 & $0.000^{\mathrm{b}}$ \\
\hline Perineural invasion & 0.475 & 0.223 & 4.514 & 1.608 & 1.037 & 2.491 & $0.034^{\mathrm{a}}$ \\
\hline $\mathrm{LN}$ metastasis & 0.641 & 0.225 & 8.098 & 1.899 & 1.221 & 2.954 & $0.004^{\mathrm{b}}$ \\
\hline DEK & 0.824 & 0.232 & 12.639 & 2.280 & 1.447 & 3.591 & $0.000^{\mathrm{b}}$ \\
\hline \multicolumn{8}{|c|}{ Multivariate survival analyses } \\
\hline Tumor size & 0.476 & 0.244 & 3.799 & 1.609 & 0.997 & 2.596 & 0.051 \\
\hline Histological grade & 0.588 & 0.180 & 10.697 & 1.801 & 1.266 & 2.563 & $0.001^{\mathrm{b}}$ \\
\hline TNM stage & 1.222 & 0.265 & 21.213 & 3.396 & 2.018 & 5.713 & $0.000^{\mathrm{b}}$ \\
\hline Perineural invasion & 0.428 & 0.255 & 2.816 & 1.534 & 0.931 & 2.528 & 0.093 \\
\hline LN metastasis & 0.259 & 0.267 & 0.941 & 1.296 & 0.768 & 2.187 & 0.332 \\
\hline DEK & 0.719 & 0.238 & 9.110 & 2.023 & 1.287 & 3.274 & $0.003^{\mathrm{b}}$ \\
\hline
\end{tabular}

Statistical analyses were performed using Cox proportional hazard regression model; ${ }^{\mathrm{a}} \mathrm{P}<0.05$ and ${ }^{\mathrm{b}} \mathrm{P}<0.01$. PDACs, pancreatic ductal adenocarcinomas. CI, confidence interval; HR, hazard ratio; TNM, tumor-node-metastasis; LN, lymph node.

with OS in patients with PDAC (Table III), indicating DEK overexpression may be a valuable prognostic factor for PDAC. Therefore, further multivariate analysis was performed for all of the significant variables examined in the univariate analysis. These data suggest that DEK overexpression [hazard ratio (HR), 2.023; 95\% confidence interval (CI), 1.287-3.274; $\mathrm{P}=0.003$ ], histological grade (HR, 1.801; 95\% CI, 1.266-2.563; $\mathrm{P}=0.001)$ and TNM stage (HR, 3.396; 95\% CI, 2.018-5.713; $\mathrm{P}=0.000)$ proved to be independent prognostic factors in prognosis of PDAC. To further substantiate the importance of DEK overexpression in PDAC progression, we analyzed the association between DEK expression and OS of 87 PDAC cases using the Kaplan-Meier method. OS rates were significantly higher in PDAC cases with DEK low-expression than in those with DEK overexpression (Fig. 5A). Combination analysis showed that DEK overexpression influenced OS rates of PDAC in grade 1 and 2, and early-stage (I-II) groups ( $\log$-rank=6.303, 6.014 and 11.865, respectively; $\mathrm{P}=0.012,0.014$ and 0.001 , respectively) (Fig. 5B, C and E). However, in the groups of patients with grade 3 and late-stage tumors (III-IV), the OS rate was not correlated with DEK expression status (log-rank=3.299 and 2.553, respectively; $\mathrm{P}=0.069$ and 0.110 , respectively) (Fig. 5D and $\mathrm{F}$ ).

\section{Discussion}

Pancreatic ductal adenocarcinoma (PDAC), a frequent and challenging tumor, is a deadly disease with a dismal prognosis. The characteristics of PDAC include an aggressive rate of tumor growth and high incidence of metastasis (25).
Currently, the most patients are in an advanced or metastatic condition at the time of diagnosis, and only $\sim 15 \%$ of cases can be surgically removed (3). Zhou et al reported that the median survival time of patients with PDAC was only 13.4 months after curative resection (26). Therefore, the identification of a sensitive and reliable biomarker for the early detection of PDAC is greatly needed. In the recent study, we evaluated the clinicopathological value of DEK overexpression in patients with PDAC.

DEK, a transcription factor, is a conserved non-histone nucleoprotein without known paralogs $(27,28)$. The human DEK gene is an important proto-oncogene that is involved in a variety of tumor-associated transcriptional and post-translational modifications (29). Numerous studies have shown that DEK functions as a positive supporting transcriptional factor to induce the expression of target genes. Sawatsubashi et al showed that DEK was correlated with numerous transcriptionally active areas of chromatin and the nuclear ecdysone receptor, exerting its functions as a transcriptional activator in Drosophila (30). Sandén et al found that DEK preferentially bound to regions of euchromatin near the transcription start sites of highly expressed genes in lymphoma cells and was involved with common transcriptional regulators including SP1 and RNA polymerase II (31). Vinnedge et al showed that DEK drove the expression of Wnt ligands, enhancing $\beta$-catenin transcriptional activity in breast cancer cells $(32,33)$. Adams et al reported that DEK can activate transcription via interaction with IRAK1 in head and neck cancer (34). These findings indicate that DEK potentially plays important roles in the progression of tumor cells. 

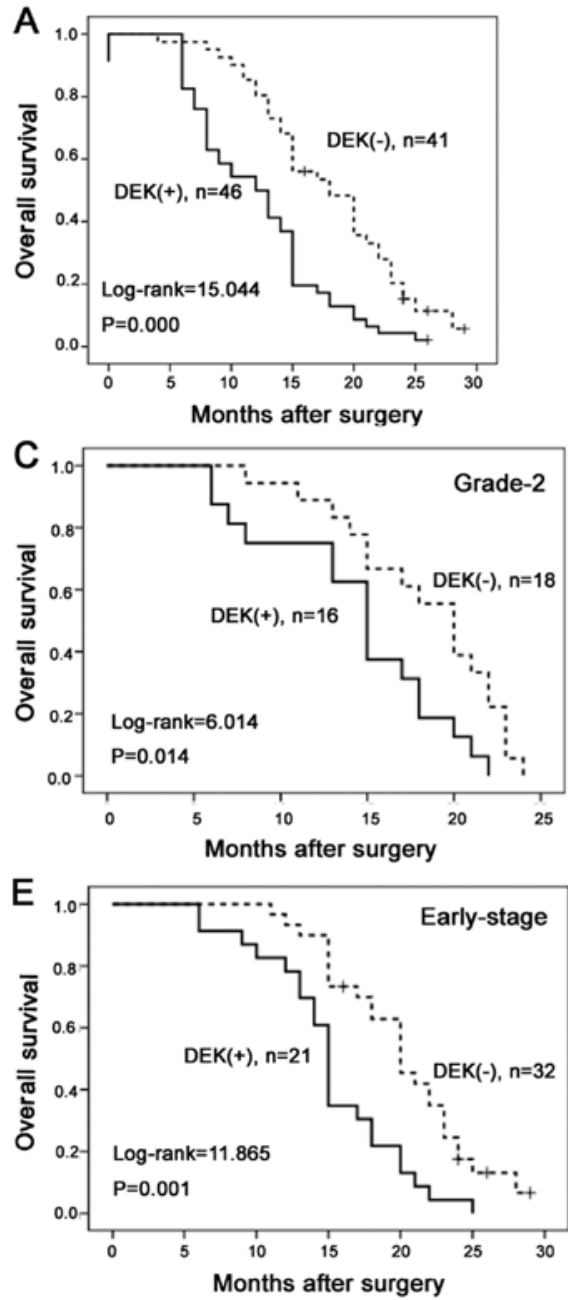
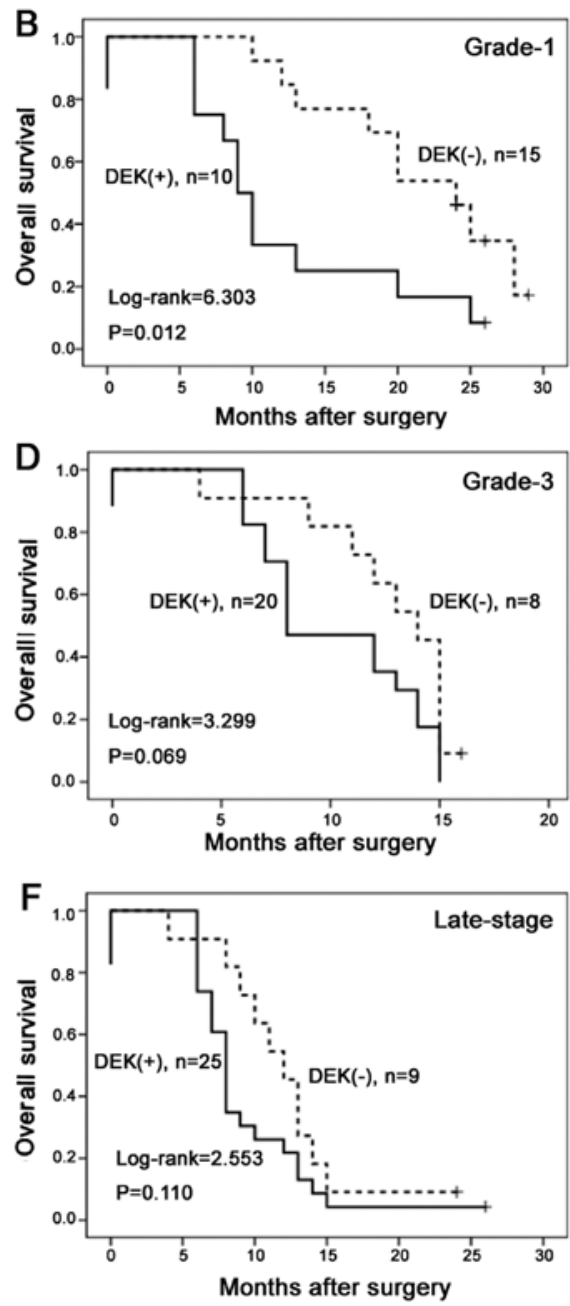

Figure 5. Kaplan-Meier analyses of OS rates in 87 PDAC patients in relation to DEK protein overexpression. (A) PDAC patients with DEK overexpression had a lower OS rate than those with DEK low-expression (log-rank=15.044, $\mathrm{P}=0.000)$. ( $\mathrm{B}, \mathrm{C}$ and E) PDAC patients with DEK overexpression in grade 1 and 2 , and early-stage groups had significantly lower OS rates than those with DEK low-expression (log-rank=6.303, 6.041 and 11.865, respectively; $\mathrm{P}=0.012,0.014$ and 0.001 , respectively). (D and F) The OS rates for DEK expression did not show any differences in grade 3 and late-stage groups.

Recently, Datta et al validated that the level of DEK expression was markedly higher in bladder cancer than normal counterparts using western blotting, suggesting that DEK may be a biomarker for the detection of bladder cancer (35). In the present study, our principal aim was to determine whether DEK overexpression is a biomarker for the prognostic evaluation of PDAC. In the present study, we assessed DEK mRNA expression in PDAC clinical samples using microarray data from GEO, and performed Immunoenzyme staining of DEK in 87 PDAC tissues and 52 adjacent normal pancreas tissues. We found that the expression levels of DEK mRNA in tumor tissues were significantly higher than that in the adjacent non-tumor tissues. Simultaneously, the DEK protein gave a primarily nuclear staining pattern based on immunoenzyme staining, which was consistent with Kappes et al and our IF staining results for PANC-1 pancreatic cancer (PC) cells (29). In the present study, using immunoenzyme staining of the DEK protein, we found that the DEK protein was highly expressed in PDAC tissues, while the staining was weak positive or negative in normal tissues. These findings demonstrated that DEK may play an important role in the progression and aggressiveness of PDAC.
Despite the significant association between DEK overexpression and numerous types of cancers, studies of DEK expression-based outcome in patients are limited. Liu et al demonstrated a significant association between DEK overexpression and poor survival of non-small cell lung carcinoma patients (36). Shibata et al showed that DEK overexpression was associated with tumor initiation activity and a poor prognosis in high grade neuroendocrine carcinoma of the lung (13). Our previous study reported that DEK overexpression was not only strongly associated with breast cancer, but that the expression was also higher in high grade breast cancers, as well as advanced stage tumors (23). In the present study, we also found that DEK overexpression was significantly correlated with tumor size $(\mathrm{P}=0.001)$, histological grade $(\mathrm{P}=0.050)$ and TNM stage $(\mathrm{P}=0.002)$. Unfortunately, high histological grade and advanced TNM stage indicate poor outcomes and recurrence in patients with PDAC. Therefore, DEK protein may be a novel biomarker related to progression and aggressiveness of PDAC.

In regards to survival rates, we found that the level of DEK expression was strongly correlated with the survival rates in patients with PDAC. Additionally, univariate survival analysis 
showed that tumor size, histological grade, TNM stage, perineural invasion status and LN metastasis were all associated with OS rates in patients with PDAC. Multivariate survival analysis revealed that DEK overexpression was an independent prognostic factor along with histological grade and TNM stage. Furthermore, combination analysis showed that DEK overexpression influenced OS rates of PDAC in grade 1 and 2, and early-stage groups. However, in the groups of patients with grade 3 and late-stage tumors, the OS rate was not correlated with DEK expression status. Apparently, these findings indicated that DEK may be a potentially predictive biomarker of poor prognosis, particularly in patients with low histological grade and early-stage PDAC.

In conclusion, DEK plays an important role in the progression of PDAC. Its overexpression may be associated with PDAC progression, and may be used as a biomarker for prognostic evaluation and as a therapeutic target in PDAC. Further studies are required to confirm this hypothesis using molecular biology experiments.

\section{Acknowledgements}

The present study was supported by grants from the Special Research Project of the '973 Plan' (2014CB560708), the National Natural Science Funds of China (no. 61371067), and the International Cooperation Project of Science and Technology Department of Jilin Province (no. 20150414030GH).

\section{References}

1. Urayama S: Pancreatic cancer early detection: Expanding higher-risk group with clinical and metabolomics parameters. World J Gastroenterol 21: 1707-1717, 2015.

2. Vernejoul F, Faure P, Benali N, Calise D, Tiraby G, Pradayrol L, Susini C and Buscail L: Antitumor effect of in vivo somatostatin receptor subtype 2 gene transfer in primary and metastatic pancreatic cancer models. Cancer Res 62: 6124-6131, 2002.

3. Recaldini C, Carrafiello G, Bertolotti E, Angeretti MG and Fugazzola C: Contrast-enhanced ultrasonograpic findings in pancreatic tumors. Int J Med Sci 5: 203-208, 2008.

4. Stathis A and Moore MJ: Advanced pancreatic carcinoma: Current treatment and future challenges. Nat Rev Clin Oncol 7: 163-172, 2010.

5. Lee SH, Kim H, Hwang JH, Lee HS, Cho JY, Yoon YS and Han HS: Breast cancer resistance protein expression is associated with early recurrence and decreased survival in resectable pancreatic cancer patients. Pathol Int 62: 167-175, 2012.

6. Liu K, Ji B, Zhang W, Liu S, Wang Y and Liu Y: Comparison of iodine-125 seed implantation and pancreaticoduodenectomy in the treatment of pancreatic cancer. Int J Med Sci 11: 893-896, 2014.

7. Yamashita K, Miyamoto A, Hama N, Asaoka T, Maeda S, Omiya H, Takami K, Doki Y, Mori M and Nakamori S: Survival impact of pulmonary metastasis as recurrence of pancreatic ductal adenocarcinoma. Dig Surg 32: 464-471, 2015.

8. Van den Broeck A, Vankelecom H, Van Eijsden R, Govaere O and Topal B: Molecular markers associated with outcome and metastasis in human pancreatic cancer. J Exp Clin Cancer Res 31: 68, 2012.

9. Niccolai E, Cappello P, Taddei A, Ricci F, D'Elios MM, Benagiano M, Bechi P, Bencini L, Ringressi MN, Coratti A, et al: Peripheral ENO1-specific T cells mirror the intratumoral immune response and their presence is a potential prognostic factor for pancreatic adenocarcinoma. Int J Oncol 49: 393-401, 2016.

10. Ma C, Nong K, Wu B, Dong B, Bai Y, Zhu H, Wang W, Huang X, Yuan Z and Ai K: miR-212 promotes pancreatic cancer cell growth and invasion by targeting the hedgehog signaling pathway receptor patched-1. J Exp Clin Cancer Res 33: 54, 2014.
11. von Lindern M, Fornerod M, van Baal S, Jaegle M, de Wit T, Buijs A and Grosveld G: The translocation (6;9), associated with a specific subtype of acute myeloid leukemia, results in the fusion of two genes, dek and can, and the expression of a chimeric, leukemia-specific dek-can mRNA. Mol Cell Biol 12: 1687-1697, 1992.

12. Khodadoust MS, Verhaegen M, Kappes F, Riveiro-Falkenbach E, Cigudosa JC, Kim DS, Chinnaiyan AM, Markovitz DM and Soengas MS: Melanoma proliferation and chemoresistance controlled by the DEK oncogene. Cancer Res 69: 6405-6413, 2009.

13. Shibata T, Kokubu A, Miyamoto M, Hosoda F, Gotoh M, Tsuta K, Asamura H, Matsuno Y, Kondo T, Imoto I, et al: DEK oncoprotein regulates transcriptional modifiers and sustains tumor initiation activity in high-grade neuroendocrine carcinoma of the lung. Oncogene 29: 4671-4681, 2010.

14. Sammons M, Wan SS, Vogel NL, Mientjes EJ, Grosveld G and Ashburner BP: Negative regulation of the RelA/p65 transactivation function by the product of the DEK proto-oncogene. J Biol Chem 281: 26802-26812, 2006.

15. Gamble MJ and Fisher RP: SET and PARP1 remove DEK from chromatin to permit access by the transcription machinery. Nat Struct Mol Biol 14: 548-555, 2007.

16. Wise-Draper TM, Morreale RJ, Morris TA, Mintz-Cole RA, Hoskins EE, Balsitis SJ, Husseinzadeh N, Witte DP, WikenheiserBrokamp KA, Lambert PF, et al: DEK proto-oncogene expression interferes with the normal epithelial differentiation program. Am J Pathol 174: 71-81, 2009.

17. Kappes F, Fahrer J, Khodadoust MS, Tabbert A, Strasser C, Mor-Vaknin N, Moreno-Villanueva M, Bürkle A, Markovitz DM and Ferrando-May E: DEK is a poly(ADP-ribose) acceptor in apoptosis and mediates resistance to genotoxic stress. Mol Cell Biol 28: 3245-3257, 2008

18. Kroes RA, Jastrow A, McLone MG, Yamamoto H, Colley P, Kersey DS, Yong VW, Mkrdichian E, Cerullo L, Leestma J, et al: The identification of novel therapeutic targets for the treatment of malignant brain tumors. Cancer Lett 156: 191-198, 2000.

19. von Lindern M, van Baal S, Wiegant J, Raap A, Hagemeijer A and Grosveld G: can, a putative oncogene associated with myeloid leukemogenesis, may be activated by fusion of its $3^{\prime}$ half to different genes: Characterization of the set gene. Mol Cell Biol 12: 3346-3355, 1992.

20. Sanchez-Carbayo M,SocciND,Lozano JJ,LiW,CharytonowiczE, Belbin TJ, Prystowsky MB, Ortiz AR, Childs G and CordonCardo C: Gene discovery in bladder cancer progression using cDNA microarrays. Am J Pathol 163: 505-516, 2003.

21. Kondoh N, Wakatsuki T, Ryo A, Hada A, Aihara T, Horiuchi S, Goseki N, Matsubara O, Takenaka K, Shichita M, et al: Identification and characterization of genes associated with human hepatocellular carcinogenesis. Cancer Res 59: 4990-4996, 1999.

22. Lin L, Piao J, Gao W, Piao Y, Jin G, Ma Y, Li J and Lin Z: DEK over expression as an independent biomarker for poor prognosis in colorectal cancer. BMC Cancer 13: 366, 2013.

23. Liu S, Wang X, Sun F, Kong J, Li Z and Lin Z: DEK overexpression is correlated with the clinical features of breast cancer. Pathol Int 62: 176-181, 2012.

24. Piao J, Shang Y, Liu S, Piao Y, Cui X, Li Y and Lin Z: High expression of DEK predicts poor prognosis of gastric adenocarcinoma. Diagn Pathol 9: 67, 2014.

25. Chatterjee D, Katz MH, Rashid A, Wang H, Iuga AC, Varadhachary GR, Wolff RA, Lee JE, Pisters PW, Crane CH, et al: Perineural and intraneural invasion in posttherapy pancreaticoduodenectomy specimens predicts poor prognosis in patients with pancreatic ductal adenocarcinoma. Am J Surg Pathol 36: 409-417, 2012.

26. Zhou HY, Wang Y, Zhang J, Ruan CP, Wang WJ, Sun YP and $\mathrm{Hu} Z \mathrm{ZQ}$ : Retrograde vs conventional dissection technique in pancreaticoduodenectomy: A pilot study. JAMA Surg 149: 604-607, 2014

27. Privette Vinnedge LM, Kappes F, Nassar N and Wells SI: Stacking the DEK: From chromatin topology to cancer stem cells. Cell Cycle 12: 51-66, 2013.

28. Pease NA, Wise-Draper T and Privette Vinnedge L: Dissecting the potential interplay of DEK functions in inflammation and cancer. J Oncol 2015: 106517, 2015.

29. Kappes F, Damoc C, Knippers R, Przybylski M, Pinna LA and Gruss C: Phosphorylation by protein kinase CK 2 changes the DNA binding properties of the human chromatin protein DEK. Mol Cell Biol 24: 6011-6020, 2004. 
30. Sawatsubashi S, Murata T, Lim J, Fujiki R, Ito S, Suzuki E, Tanabe M, Zhao Y, Kimura S, Fujiyama S, et al: A histone chaperone, DEK, transcriptionally coactivates a nuclear receptor. Genes Dev 24: 159-170, 2010.

31. Sandén C, Järvstråt L, Lennartsson A, Brattås PL, Nilsson B and Gullberg U: The DEK oncoprotein binds to highly and ubiquitously expressed genes with a dual role in their transcriptional regulation. Mol Cancer 13: 215, 2014.

32. Privette Vinnedge LM, McClaine R, Wagh PK, WikenheiserBrokamp KA, Waltz SE and Wells SI: The human DEK oncogene stimulates $\beta$-catenin signaling, invasion and mammosphere formation in breast cancer. Oncogene 30: 2741-2752, 2011.

33. Privette Vinnedge LM, Benight NM, Wagh PK, Pease NA Nashu MA, Serrano-Lopez J, Adams AK, Cancelas JA, Waltz SE and Wells SI: The DEK oncogene promotes cellular proliferation through paracrine Wnt signaling in Ron receptor-positive breast cancers. Oncogene 34: 2325-2336, 2015.
34. Adams AK, Bolanos LC, Dexheimer PJ, Karns RA, Aronow BJ, Komurov K, Jegga AG, Casper KA, Patil YJ, Wilson KM, et al: IRAK1 is a novel DEK transcriptional target and is essential for head and neck cancer cell survival. Oncotarget 6: 43395-43407, 2015.

35. Datta A, Adelson ME, Mogilevkin Y, Mordechai E, Sidi AA and Trama JP: Oncoprotein DEK as a tissue and urinary biomarker for bladder cancer. BMC Cancer 11: 234, 2011.

36. Liu X, Qi D, Qi J, Mao Z, Li X, Zhang J, Li J and Gao W: Significance of DEK overexpression for the prognostic evaluation of non-small cell lung carcinoma. Oncol Rep 35: 155-162, 2016. 